\title{
LES ERREURS AFFECTANT LES PÉRIODES, LES DEMI-GRANDS AXES ET LES MASSES TOTALES \\ DES COUPLES VISUELS ORBITAUX
}

\author{
J. DOMMANGET \\ Observatoire Royal de Belgique, Bruxelles, Belgique
}

\section{Introduction}

Soit $\Delta t$, l'intervalle de temps couvert par les observations ayant permis le calcul des éléments orbitaux d'une étoile double visuelle. Soient $P$, sa période et $a^{\prime \prime}$, son demigrand axe.

Ces éléments sont affectés d'erreurs dépendant essentiellement de la précision générale du matériel d'observation, de l'importance relative $\Delta t / P$ de l'arc couvert par les mesures, de la forme et de la situation de cet arc dans l'orbite apparente. Mais on négligera ici ces deux derniers facteurs car leur considération entraîne des difficultés qu'il n'est peut-être pas nécessaire de vouloir surmonter dans une première étude.

(a) En ce qui concerne alors l'imprécision générale du matériel d'observation, on remarquera que les erreurs qui l'affectent sont de deux types:

les erreurs aléatoires à caractère local, dues aux erreurs de mesure bien connues et à la diversité des équations personnelles des observateurs;

les erreurs à caractère systématique, dues par exemple, d'une part à une mauvaise répartition des observateurs le long de l'arc d'orbite observé et à l'influence localè consécutive de leurs équations personnelles, et d'autre part, à des inégalités de densité des observations le long de ce même arc.

Ces erreurs se reportent sur les éléments orbitaux calculés à partir de ce matériel. D'ailleurs, quand on considère un couple pour lequel un grand nombre d'orbites a été calculé à partir de mesures s'étendant sur un arc d'orbite allant croissant (et par des méthodes de calcul variées) on constate que ces éléments, et plus particulièrement la période et le demi-grand axe (Figure 1), présentent en fonction du temps et dès lors en fonction de la longueur de l'are observé, des valeurs se dispersant autour de courbes moyennes tendant asymptotiquement vers les valeurs exactes de $P$ et de $a^{\prime \prime}$.

Chaque écart à la valeur asymptotique, soit pour $P$, soit pour $a^{\prime \prime}$, comporte donc une partie aléatoire (relativement à la courbe moyenne) et une partie à caractère systématique, correspondant respectivement aux deux sortes d'erreurs affectant le matériel d'observation.

Il est évident que la séparation de ces deux sortes d'erreurs n'est sans doute pas toujours aussi nette que le laisserait entendre notre façon de les présenter; mais il apparaît en tous cas, que dans une étude des erreurs affectant les éléments orbitaux des étoiles doubles visuelles, il y a lieu de considérer autant que possible ces erreurs séparément. 

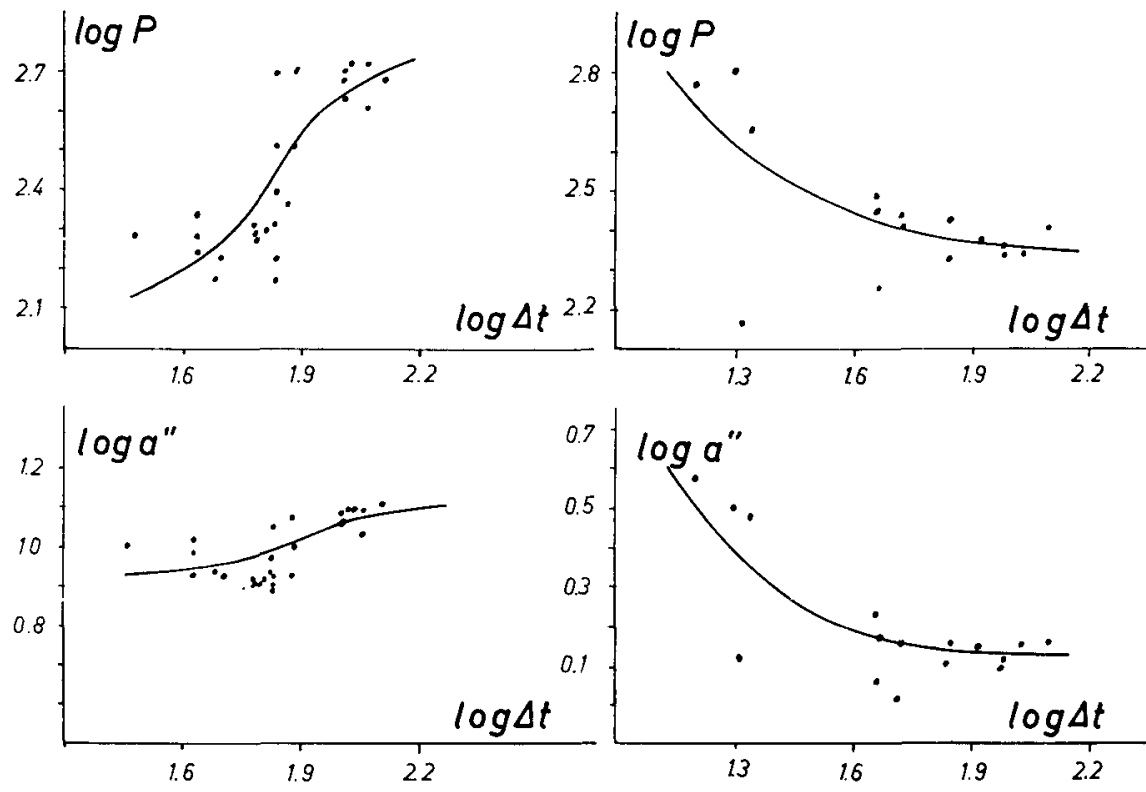

Fig. 1. Deux cas caractéristiques d'étoiles doubles visuelles (ADS 671 et ADS 9626) pour lesquelles les orbites successives témoignent à la fois d'effets systématiques et d'erreurs aléatoires locales pour les valeurs de $P$ et de $a^{\prime \prime}$.

(b) On remarquera ensuite que, pour une même importance relative $\Delta t / P$ de l'arc couvert par les observations, vis-à-vis de la période, l'erreur sur $P$ est proportionnelle à $P$, toutes autres choses étant égales, et qu'il convenait donc de considérer l'erreur sur $\log P$ en fonction de $\Delta t / P$, ou mieux, de $\log (\Delta t / P)$. Par ailleurs, on sait que l'imprécision des mesures est liée à l'importance de la séparation des composantes et dès lors approximativement, à celle du demi-grand axe. C'est pourquoi, il s'avérait intéressant de considérer également différentes classes de $a^{\prime \prime}$ ou mieux, de $\log a^{\prime \prime}$.

Quant à l'élément $a^{\prime \prime}$ lui-même, il apparaissait également préférable de considérer l'erreur sur son logarithme plutôt que sur sa valeur naturelle. En effet, pour un matériel d'observation de même précision et de même rapport $\Delta t / P$, deux orbites présentent en moyenne des demi-grands axes affectés d'erreurs qui leur sont proportionnelles, par suite du coefficient $\cos i$ existant entre l'orbite réelle et l'orbite apparente. De plus, pour des raisons d'homogénéité, nous avons considéré l'erreur sur $\log a^{\prime \prime}$ également en fonction de $\log (\Delta t / P)$ et de $\log a^{\prime \prime}$.

Par ailleurs, en ce qui concerne les erreurs affectant les masses totales déduites des orbites d'étoiles doubles visuelles, il s'imposait dès lors, de considérer l'expression logarithmique

$$
\log \mathfrak{M}_{A B}=\left(3 \log a^{\prime \prime}-2 \log P\right)-3 \log \pi^{\prime \prime},
$$

et d'étudier l'erreur qui affecte l'ensemble des deux premiers termes mis entre parenthèses, également en fonction de $\log (\Delta t / P)$ et de $\log a^{\prime \prime}$. 


\section{Matériel statistique - Calcul des erreurs moyennes (Dispersion)}

(a) Le matériel statistique a été établi en considérant pour chaque binaire individuellement, tous les groupes d'orbites constitués en considérant celles dont les époques de calcul extrêmes ne diffèrent pas de plus de 20 à $25 \%$ de la moyenne de l'intervalle de temps $\Delta t$ couvert par les observations ayant servi aux calculs de ces orbites.

Nous avons évidemment éliminé au préalable, quelques orbites manifestement fausses pour leurs époques de calcul et d'autres, présentant une indétermination de quadrant connue. Par contre, nous avons retenu quelques orbites désignées I, II, etc. par un même auteur, bien qu'elles représentent souvent des étapes de calcul.

C'est ainsi que finalement, nous avons pu disposer de quelque 573 groupes portant sur 336 couples différents et comportant au total 1671 orbites*.

(b) Pour définir facilement et avec une précision suffisante, l'intervalle de temps $\Delta t$ couvert par les observations, nous avons retenu d'une part, la date de la première observation donnée dans le catalogue Index et d'autre part, la date de publication de l'orbite. Il aurait été préférable évidemment de retourner à chacune des publications pour juger avec plus de précision des dates à considérer. Mais nous aurions eu près de 1700 articles à consulter alors que le gain de précision n'aurait pas été appréciable. Toutefois, nous sommes retourné aux sources, pour les orbites à courtes périodes (moins de 20 ans) pour lesquelles le rapport $\Delta t / P$ aurait pu être influencé trop sensiblement parfois par une erreur de plusieurs années sur $\Delta t$.

Il reste qu'il eut été peut-être également préférable, pour le calcul de $\Delta t / P$, de se servir de la valeur réelle de $P$. Mais dans la grande majorité des cas où seulement quelques orbites sont connues par couple, il est impossible de s'assurer de l'importance des effets systématiques dont il a été question plus haut et de définir les valeurs asymptotiques correspondantes les plus probables vers lesquelles tendent les éléments orbitaux et en particulier la période.

Nous nous trouvions ainsi devant un dilemme: ou bien réduire le matériel statistique aux couples dont on connaît un assez grand nombre d'orbites et pour lesquels on peut estimer avec précision, la valeur réelle de la période, mais faire perdre à ce matériel toute valeur d'échantillon représentatif et le rendre quasi inutilisable pour notre recherche, ou bien, au contraire, conserver à ce matériel toute son étendue, mais s'en tenir nécessairement aux seules valeurs de $P$ définies objectivement: leurs valeurs orbitales.

C'est cette seconde façon de faire que nous avons adoptée car, si elle entraîne sans doute une certaine déformation des résultats par la présence de cas où la période varie fort d'une orbite à la suivante - ce que nous examinerons plus loin - il ne faut pas oublier que lorsqu'on calcule une orbite d'étoile double visuelle, ce que l'on désire savoir, c'est le crédit que l'on peut accorder aux éléments orbitaux trouvés et cela, en fonction non pas de la période réelle que l'on ne connaît d'ailleurs pas, mais bien

\footnotetext{
* Ce matériel est extrait d'un fichier d'orbites établi à Uccle et comportant toutes les orbites connues
} pour chaque couple, soit quelque 2500 orbites. 


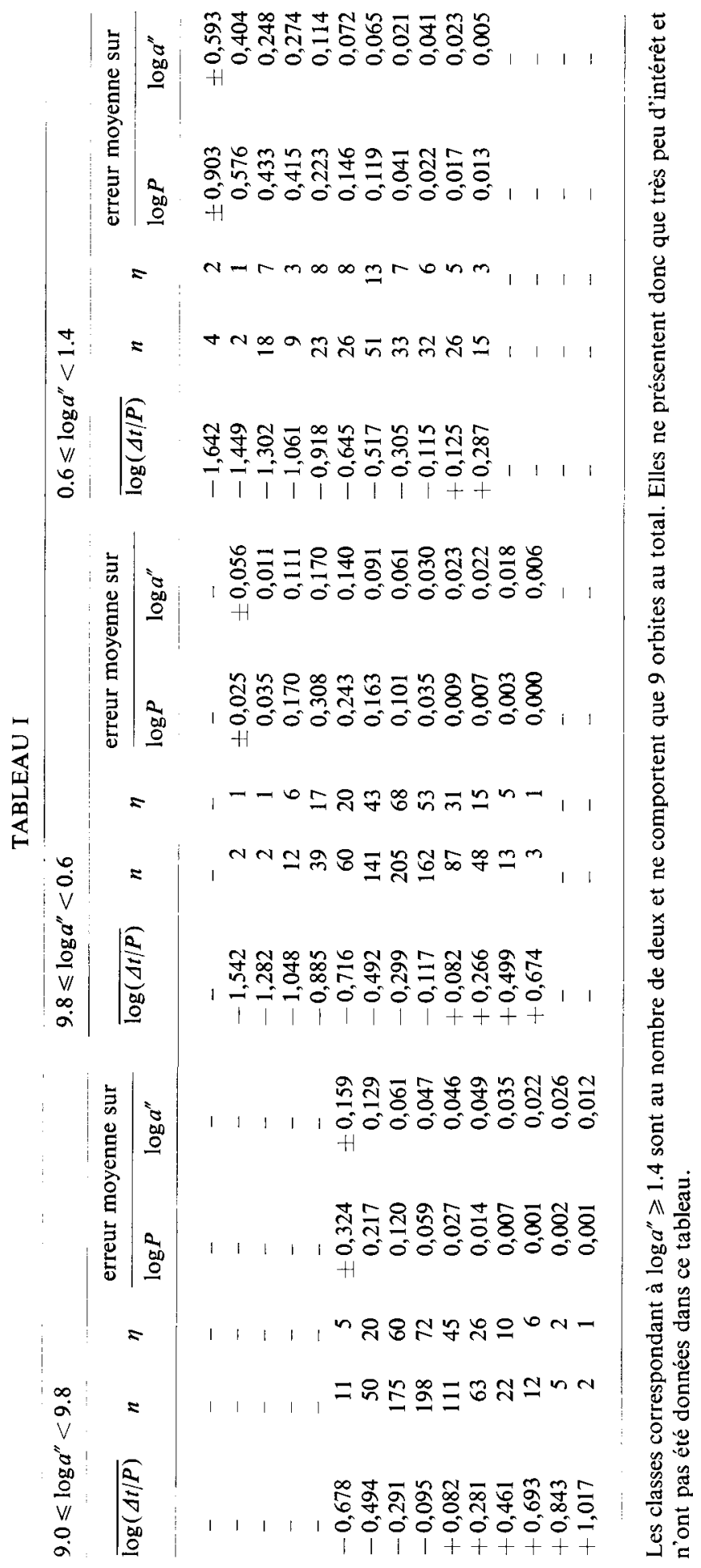




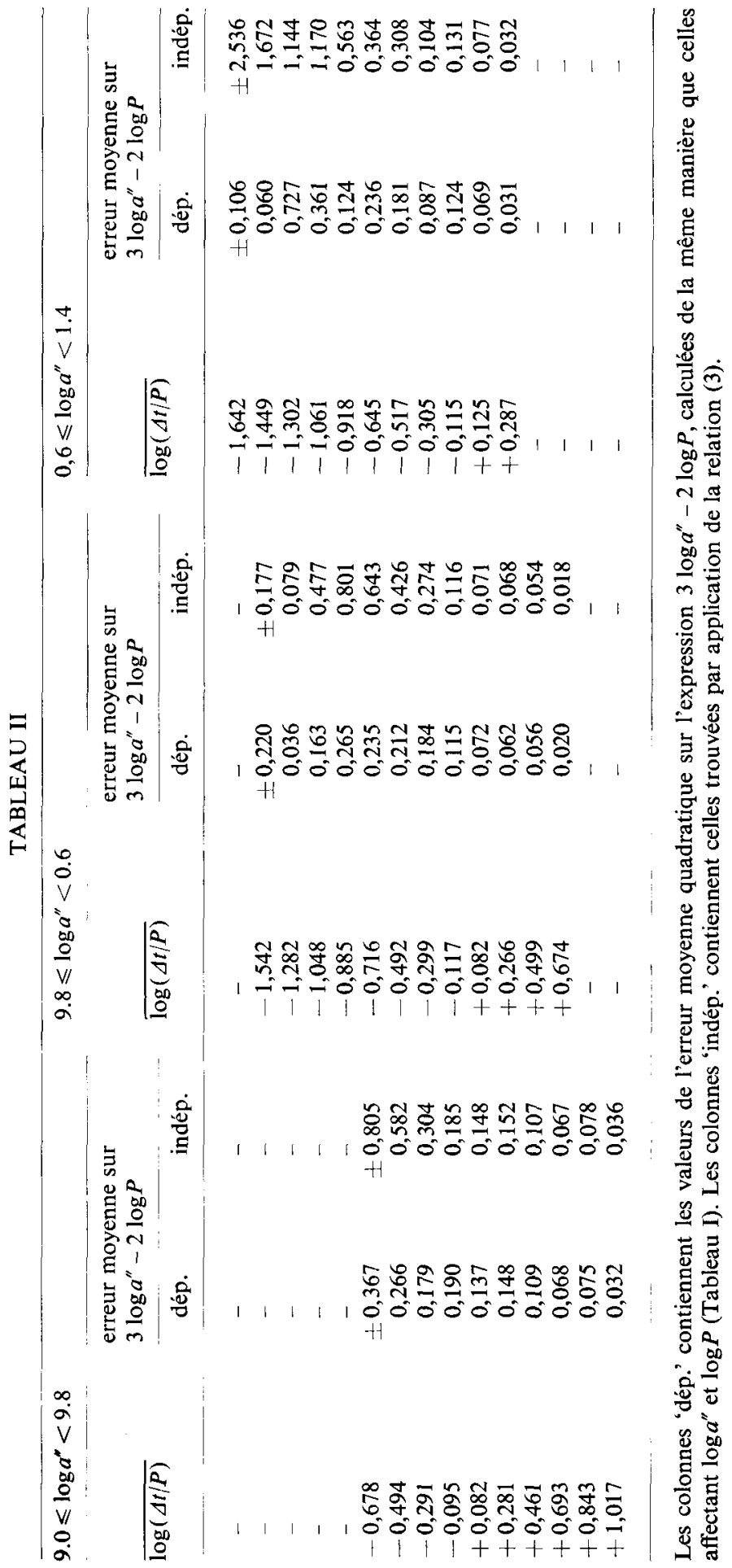


de la période calculée que l'auteur considère nécessairement, à l'époque du calcul, comme la plus valable.

Ayant fait cette option, nous avons calculé pour chacun des 573 groupes d'orbites, les valeurs moyennes de $\log P$, de $\log a^{\prime \prime}$, de $\left(3 \log a^{\prime \prime}-2 \log P\right)$ et de $\log (\Delta t / P)$. Nous les avons ensuite répartis en classes de $\log (\Delta t / P)$ et de $\log a^{\prime \prime}$, en considérant pour le premier paramètre, des intervalles de 0,2 et pour le second, des intervalles de $0,8 *$. Dans chacune des classes ainsi constituées, nous avons ensuite calculé l'erreur moyenne quadratique affectant les valeurs moyennes de $\log P, \operatorname{de} \log a^{\prime \prime}$ et de $\left(3 \log a^{\prime \prime}-2 \log P\right)$, en appliquant la relation rappelée dans notre précédente communication (voir p. 136) et où $n$ est le nombre d'orbites et $\eta$, le nombre de groupes par classe. Nous avons ainsi pu établir les Tableaux I et II.

Les graphiques des Figures 2 et 3 ne portent que sur les parties centrales des Tableaux I et II, c'est-à-dire sur celles où chaque classe contient généralement plus de $n=10$ orbites.

(c) De l'examen de la Figure 2, on peut conclure comme suit:

(1) pour toute valeur donnée de $\log (\Delta t / P)$, les erreurs moyennes quadratiques affectant $\log P$ et $\log a^{\prime \prime}$ sont d'autant plus grandes que $\log a^{\prime \prime}$ est petit, sauf peut-être, en ce qui concerne $\log P$, au delà de la valeur: $\log (\Delta t / P)=0,2$;

(2) ces erreurs décroissent rapidement quand $\log (\Delta t / P)$ augmente; elles atteignent des valeurs pratiquement constantes de l'ordre de 0,01 pour $\log P$ (soit $2,4 \%$ sur $P$ ) et de 0,01 à 0,03 pour $\log a^{\prime \prime}$ (soit de $2,4 \%$ à $7,2 \%$ sur $a^{\prime \prime}$ ), à partir d'une limite située vers

$$
\log (\Delta t / P)=0,2
$$

correspondant à

$$
\Delta t / P=1,6
$$

La Figure 3 permet une étude de la précision des valeurs déduites pour les masses totales à partir des éléments orbitaux. Une première vérification s'imposait quant au degré de dépendance des erreurs affectant la période et le demi-grand axe. Dans ce but, nous avons également calculé l'erreur moyenne quadratique qui affecterait l'expression $\left(3 \log a^{\prime \prime}-2 \log P\right.$ ) si $\log a^{\prime \prime}$ et $\log P$ étaient entachées d'erreurs aléatoires indépendantes, en utilisant la relation connue

$$
\varepsilon= \pm \sqrt{9 \varepsilon_{\log a^{\prime \prime}}^{2}+4 \varepsilon_{\log P}^{2}}
$$

où $\varepsilon_{\log a^{\prime \prime}}$ et $\varepsilon_{\log P}$ ont pour valeurs, celles données dans le Tableau I.

Le Tableau II donne à la fois les erreurs calculées sur $\left(3 \log a^{\prime \prime}-2 \log P\right)$ directement par classe d'une part et par la relation (3) d'autre part.

La Figure 3 nous apprend que:

(1) A l'erreur affectant $\log \mathfrak{M}_{A B}$ (la parallaxe étant supposée rigoureusement connue)

* D'autres intervalles de 0,2 et de 0,4 ont été considérés pour $\log a^{\prime \prime}$, mais les statistiques correspondantes ne nous ont pas paru satisfaisantes par suite du trop petit nombre d'orbites subsistant par classe. 
suit une loi semblable à celles des erreurs sur $\log a^{\prime \prime}$ et sur $\log P$; elle en possède les particularités constatées plus haut;

(2) la compensation des erreurs affectant les éléments $P$ et $a^{\prime \prime}$ dans le calcul de la masse totale est importante pour les petites valeurs de $\Delta t / P$ puisque pour $\log (\Delta t / P)=9,5$, elle correspond à une réduction de $50 \%$ sur son logarithme, mais elle va diminuant rapidement pour $\log (\Delta t / P)$ croissant;

(3) dès que $\log (\Delta t / P)$ dépasse une limite située aux environs de 0,0 (c'est-à-dire dès que $\Delta t$ couvre plus d'une période), les éléments $P$ et $a^{\prime \prime}$ sont affectés d'erreurs totalement indépendantes et cela, quel que soit le demi-grand axe;

(4) une erreur inférieure à $20 \%$ sur la masse totale n'est que très difficilement atteinte. On s'en rendra mieux compte sur le graphique de la Figure 4 donnant une représenta-
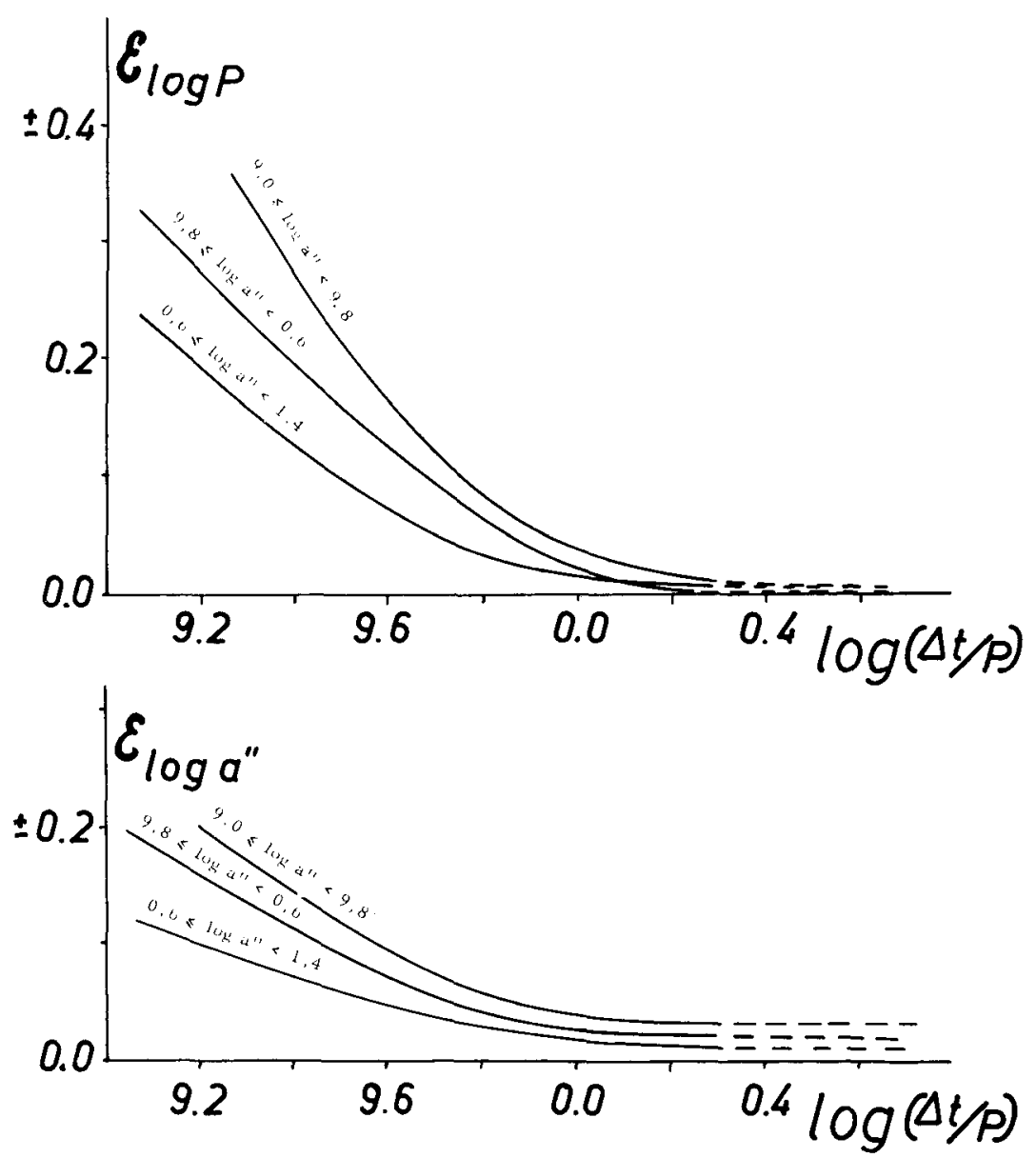

Fig. 2. Courbes moyennes établies à partir des valeurs données dans le Tableau I et représentant au mieux l'évolution des erreurs moyennes quadratiques affectant les valeurs de $\log P$ et de $\log a^{\prime \prime}$, en fonction de $\log (\Delta t / P)$ pour trois classes de $\log a^{\prime \prime}$. 

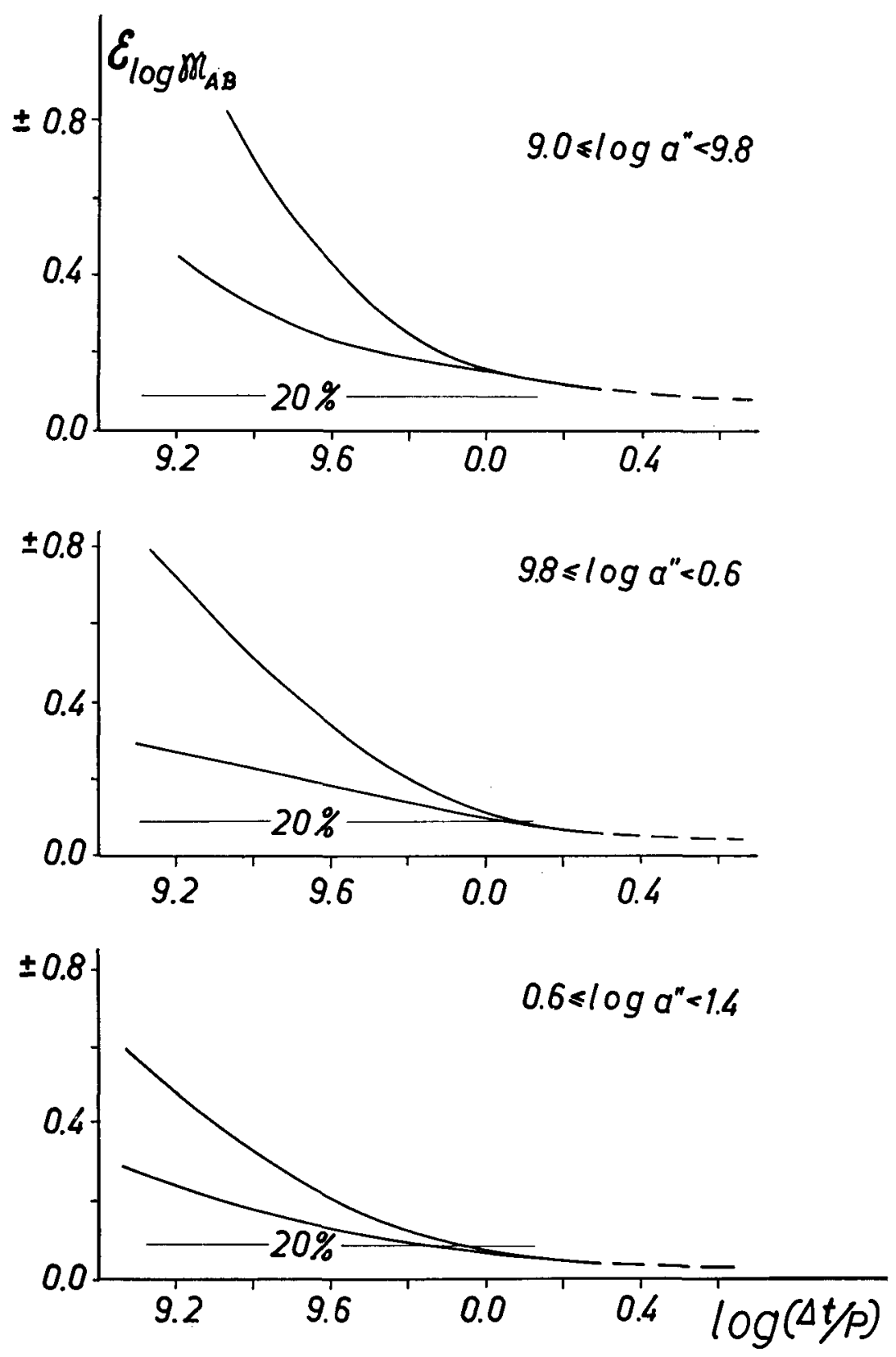

Fig. 3. Représentation graphique des valeurs données dans le Tableau II pour les erreurs affectant l'expression $\left(3 \log a^{\prime \prime}-2 \log P\right)$, d'une part telles qu'elles ressortent de notre statistique générale (courbes inférieures) et d'autre part, en la calculant sur la base d'une indépendance totale des erreurs sur les éléments $P$ et $a^{\prime \prime}$ (courbes supérieures), pour trois classes de valeurs de $\log a^{\prime \prime}$. 
tion approchée des pourcentages d'erreur sur la masse totale en fonction de $\log (\Delta t / P)$ et de $\log a^{\prime \prime}$ et établie à partir du tracé des courbes de la Figure 3.

O. J. Eggen* a également cherché à préciser la dispersion des valeurs non seulement de $P$ et de $a^{\prime \prime}$, mais surtout de la masse totale de certains couples dont il s'est servi

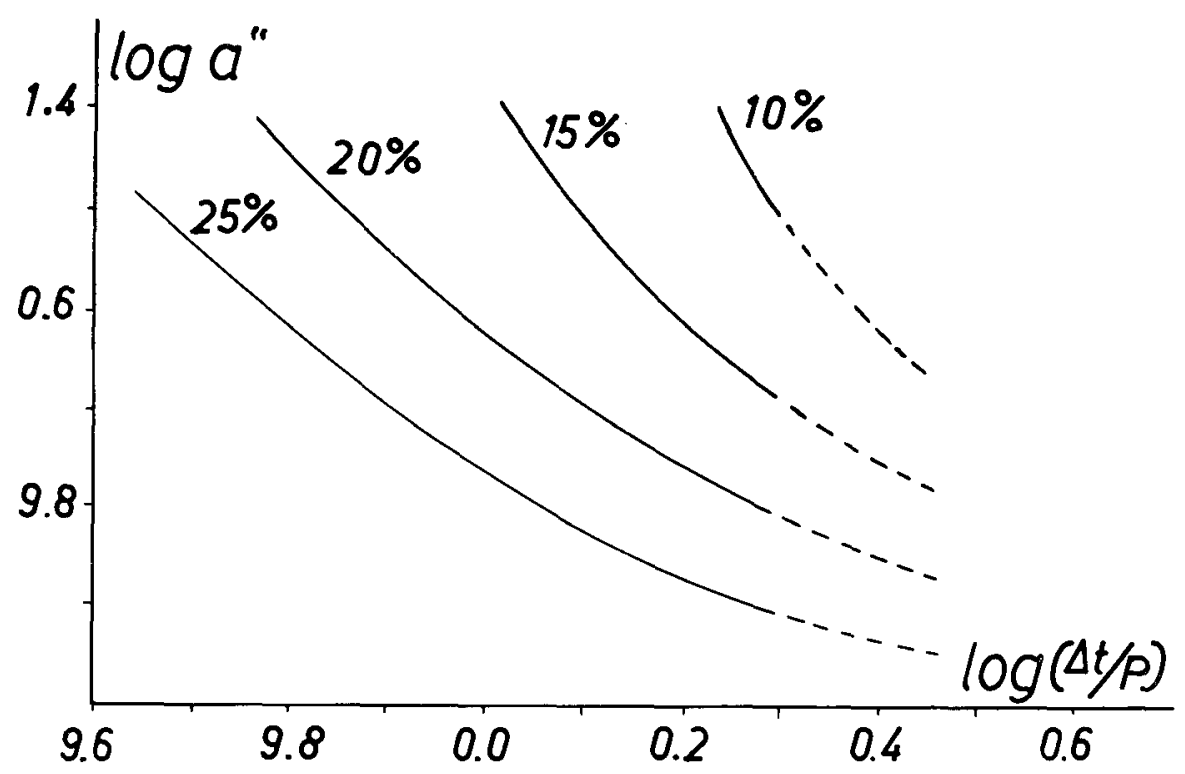

Fig. 4. Représentation approchée du pourcentage d'erreur sur la masse totale d'un couple, calculée à partir de la troisième loi de Kepler.

pour l'étude de la relation mass-luminosité, en considérant pour chacun d'eux, diverses orbites également représentatives du matériel disponible à l'époque. Des écarts assez sensibles apparaissent également sur la plupart des masses ainsi déterminées.

Quant aux conséquences du choix des valeurs orbitales individuelles de $P$ en lieu et place de leurs valeurs réelles, elles n'altèrent pratiquement pas nos conclusions. En effet, une erreur sensible sur la période entraîne dans certains cas, le passage de l'orbite correspondante d'une des classes considérées en $\log (\Delta t / P)$ à la classe voisine. De tels passages n'ont lieu que rarement et pour des valeurs de $P$ très mal définies c'est-à-dire - ainsi que nous venons de le voir à posteriori - pour des valeurs de log $(\Delta t / P)$ toujours beaucoup plus petites que 0 , c'est-à-dire pour des intervalles de temps $\Delta t$, très inférieurs aux périodes réelles elles-mêmes.

L'allure générale des courbes des Figures 2 et 3 ne peut donc s'en trouver très sensiblement altérée et ne l'est certainement que de manière insignifiante pour des valeurs de $\log (\Delta t / P)$ dépassant 9,8 à 9,9 , c'est-à-dire pour des régions sur lesquelles portent nos principales conclusions.

* The Astronomical Journal 70, 1965, p. 19.

Annual Review of Astronomy and Astrophysics 5, 1967, p. 105. 


\section{Les erreurs systématiques}

Du matériel statistique utilisé au paragraphe précédent, nous avons extrait tous les couples pour lesquels une dizaine d'orbites au moins ont été calculées. Quelque 58 cas ont ainsi été sélectionnés. Pour chacun d'eux, nous avons dressé les graphiques donnant $\log P, \log a^{\prime \prime}$ et $\left(3 \log a^{\prime \prime}-2 \log P\right)$ en fonction $\operatorname{de} \log \Delta t$.

On remarquera que nous n'avons pas considéré ici en abscisses, $\log (\Delta t / P)$ pour éviter de faire intervenir une valeur quelconque de $P$. Alors que dans l'étude de l'erreur moyenne quadratique, nous avons vu que des valeurs de $P$ systématiquement trop grandes ou trop courtes étaient susceptibles de faire passer d'une classe à l'autre de notre statistique un groupe d'orbites calculées à une même époque sans toutefois altérer sensiblement l'erreur moyenne correspondante, dans le cas présent, où précisément, il y a lieu de mettre en évidence les effets systématiques sur la période, sur le demigrand axe et sur l'expression $\left(3 \log a^{\prime \prime}-2 \log P\right)$, il s'avérait indispensable de se libérer de toute influence des erreurs systématiques sur $P$.

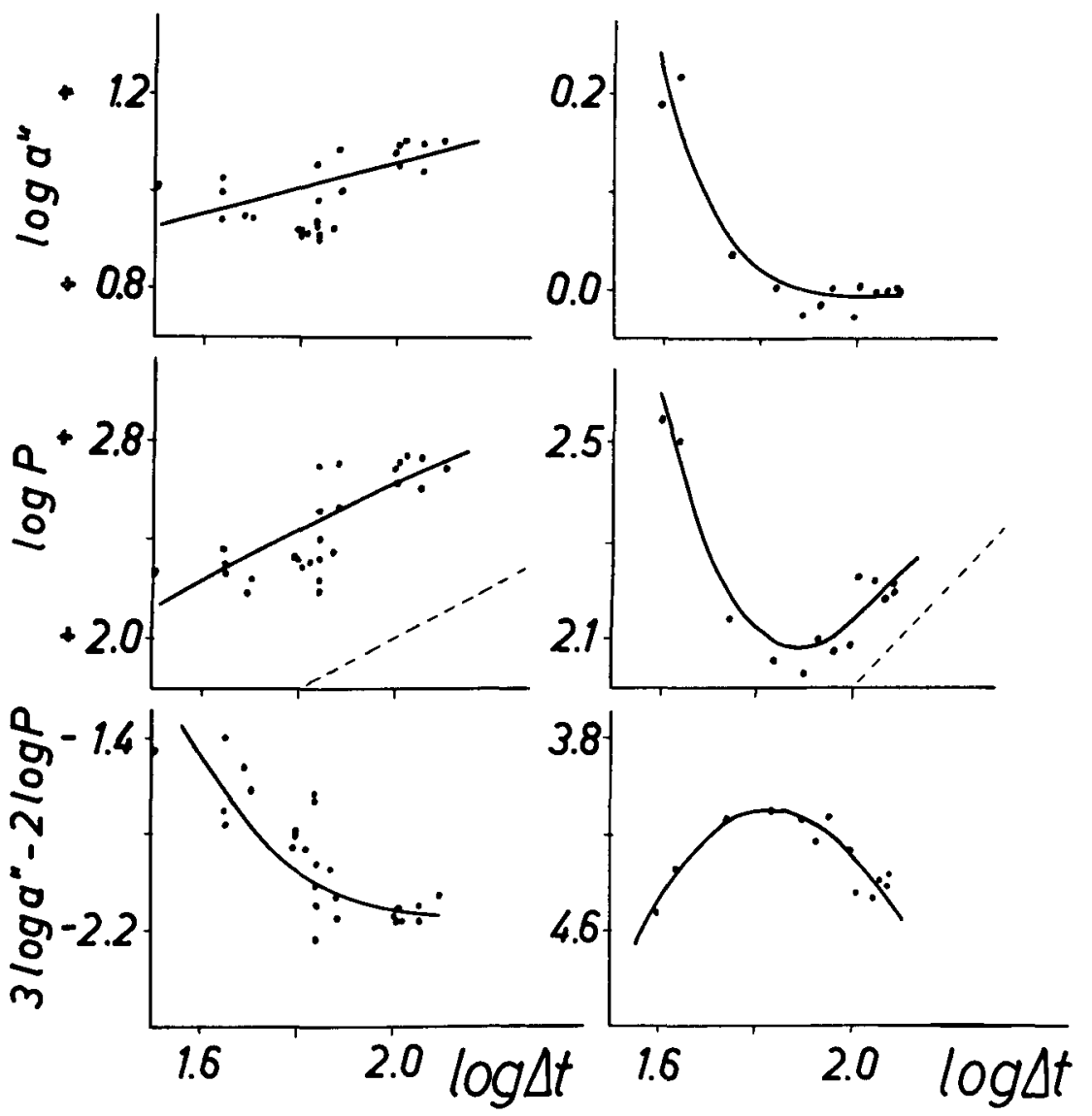

Fig. 5. Erreurs systématiques affectant les éléments orbitaux des couples ADS 102 et ADS 755. 
A cette occasion, nous présenterons tout d'abord deux cas permettant de mieux faire comprendre notre point de vue. Ils sont donnés à la Figure 5. Pour ADS 102, on remarquera que l'effet systématique sur la période correspond à un accroissement de $\log P$ égal à celui de $\log \Delta t$. Dans ces conditions, la considération en abscisses de la grandeur $\log (\Delta t / P)$ reviendrait à un regroupement de tous les points représentatifs des orbites autour de la valeur $\log (\Delta t / P)=9,4$ et à une disparition apparente de tout effet systématique. Dans le cas de ADS 755, le phénomène systématique est plus curieux encore : après une décroissance assez régulière des valeurs de $P$, une augmentation tout aussi régulière a suivi. Il est évident que dans chacun de ces deux cas, la période ne pourra croître indéfiniment avec l'accroissement du matériel d'observation et qu'il arrivera une époque où la stabilisation s'amorcera, au plus tard lorsque $\Delta t$ sera voisin de $P$. Mais il est évidemment impossible, à partir des graphiques de la Figure 5 de prévoir les valeurs correspondantes de $\log (\Delta t)$ et dès lors aussi des périodes.

Les deux cas présentés ici sont heureusement relativement rares et généralement, l'évolution de $\log P$ est beaucoup moins rapide que celle de $\log (\Delta t)$, ou même, est de sens opposé.

Quant à l'exploitation des graphiques établis pour les 58 cas considérés, il apparaît que leur diversité d'aspect ne permet pas de procéder à un dépouillement systématique des phénomènes observés. Mais de l'examen de l'ensemble, il se dégage toutefois une série de constatations intéressantes:

(1) l'effet systématique en $\log a^{\prime \prime}$ et celui en $\log P$ sont:

(a) de même sens, dans 27 , soit $46 \%$ des cas;

(b) sont insignifiants pour l'un des paramètres au moins, dans 23 , soit $40 \%$ des cas;

(c) sont de sens opposés, dans 8 , soit $14 \%$ des cas.

(2) Les effets systématiques sont généralement absorbés par les erreurs moyennes quadratiques, à partir de $\log (\Delta t / P) \approx 0,0$.

(3) Même dans les cas où les effets en $\log P$ et en $\log a^{\prime \prime}$ sont de même sens, la compensation des erreurs laisse généralement subsister sur l'expression $\left(3 \log a^{\prime \prime}-2 \log P\right)$, un effet systématique résiduel sensible. Cet effet est généralement positif. En effet sur les 58 cas, on a dénombré:

39 cas, soit $67 \%$ où l'erreur systématique est positive,

11 cas, soit $19 \%$ où l'erreur systématique est négative et

8 cas, soit $14 \%$ où l'erreur systématique ne peut être appréciée.

En sélectionnant les 24 couples pour lesquels cet effet systématique est bien défini dans l'intervalle $(9.6-0,0)$ de $\log (\Delta t / P)$ nous avons trouvé que cet effet est en moyenne de

$$
+0,10 \pm 0,22
$$

pour $\log (\Delta t / P)=9,6$.

\section{Conclusions et remarques générales}

Il résulte de cette étude que seuls les couples pour lesquels les observations couvrent un peu plus d'une période de révolution ou moins, devraient être pris en considération pour la détermination des masses totales. Car sous cette condition: 
(a) on peut déterminer facilement les valeurs les plus probables des périodes et des demi-grands axes, les erreurs qui les affectent ne comportant pas de composantes systématiques; ces erreurs sont uniquement aléatoires et, par ailleurs, stochastiquement indépendantes;

(b) les erreurs moyennes quadratiques sont inférieures à $\pm 0,1$, sur l'expression $3 \log a^{\prime \prime}-2 \log P$, soit inférieures à 20 à $25 \%$ sur $a^{\prime \prime 3} / P^{2}$.

Toutefois, le calcul des masses des binaires se présente sous deux aspects distincts suivant les buts poursuivis et exige, suivant les cas, une considération assez différente.

En effet, quand on désire procéder à une étude de la relation mass-luminosité, on se sert exclusivement de la troisième loi de Kepler pour calculer les masses totales. D'après sa forme logarithmique (1), l'erreur d'origine orbitale affectant $\log \mathfrak{M}_{A B}$ est celle de l'expression

$$
3 \log a^{\prime \prime}-2 \log P .
$$

A celle-ci s'ajoute bien entendu, l'erreur entachant les valeurs observées de $\pi$ ", laquelle est toutefois, pour les binaires proches, inférieure à $10 \%$, c'est-à-dire inférieure à 0,04 , sur $\log \pi^{\prime \prime}$.

Par contre, si pour la valeur de la parallaxe, on adopte celle de la parallaxe dynamique basée elle-même sur la relation masse-luminosité dont l'expression générale est

$$
\log \mathfrak{M}=-\alpha\left(M_{\mathrm{bol}}-\beta\right),
$$

la relation (1) devient

$$
\log \mathfrak{M}_{A B}=-\frac{5 \alpha}{3-5 \alpha}\left(3 \log a^{\prime \prime}-2 \log P\right)-\frac{3 \alpha}{3-5 \alpha} m_{A}^{\prime}+\frac{3}{3-5 \alpha} \log \mu,
$$

où

$$
m_{A}^{\prime}=m_{v A}+C_{A}+5-\beta,
$$$$
\log \mu=\text { fonction de } \Delta m^{\prime} \text { uniquement } *
$$

et où, d'après P. Baize, $\alpha=+0,1117$ et $\beta=+4,77$. Avec ces valeurs, (5) s'écrit

$$
\log \mathfrak{M}_{A B}=-0,22875\left(3 \log a^{\prime \prime}-2 \log P\right)-0,1373 m_{A}^{\prime}+1,2288 \log \mu,(6)
$$

et l'on constate que l'erreur sur $\log \mathfrak{M}_{A B}$ est, par cette relation, $1 / 0,23=4,4$ fois moins sensible aux erreurs orbitales que par l'emploi de la relation (1). A ces erreurs, il faut évidemment ajouter celles affectant $m_{v A}$ et $C_{A}$, ainsi que les erreurs affectant les paramètres $\alpha$ et $\beta$ de la relation masse-luminosité.

Mais de toute façon, on peut conclure que pour une même imprécision totale de 0,1 environ sur la détermination de $\log \mathfrak{M}_{A B} \operatorname{par}(1)$ et par (6), on peut se permettre de choisir des orbites moins bien assurées dans le cas des parallaxes dynamiques que dans celui des parallaxes observées. Ceci signifie que l'on peut dès lors, pour des recherches statistiques de divers ordres n'ayant aucun rapport avec l'établissement de la relation masse-luminosité, retenir un plus grand nombre de couples en choisissant par exemple,

* Critère de non-périodicité d'un couple stellaire. - Bulletin Astronomique 20, 1955, Tableau II, p. 11. 
pour $\log (\Delta t / P)$, une limite située vers 9,6 (Figure 3) au lieu de $0,0,1$ 'erreur quadratique moyenne étant alors de 0,2 à 0,3 sur l'expression $3 \log a^{\prime \prime}-2 \log P$ et l'erreur systématique, de 0,1 en moyenne, c'est-à-dire respectivement de moins de 0,1 et de 0,025 sur le terme orbital de l'expression (6).

Nous terminerons cette étude en remarquant qu'elle a été possible grâce au grand nombre d'orbites dont nous avons pu disposer. On a souvent critiqué (van den Bos*) le fait que plusieurs auteurs procèdent au calcul de l'orbite d'une même binaire sur la base d'un matériel quasi-identique, sous prétexte que l'orbite étant bien assurée par exemple, on assiste alors à un gaspillage d'énergie. En fait, seule l'intercomparaison d'orbites calculées par divers auteurs permet une estimation correcte de l'imprécision des éléments orbitaux et de leur valeur. Quant à l'existence d'effets systématiques, on ne peut en estimer l'importance ni le sens, tant qu'un ensemble d'orbites calculées successivement sur un assez long intervalle de temps ne soit disponible ou bien que le couple n'ait effectué une révolution complète depuis sa découverte.

Personnellement nous souhaitons que de telles pratiques se poursuivent, mais bien entendu dans une mesure raisonnable.

\section{Remerciements}

Il nous reste à remercier M. O. Nys, Calculateur à l'Observatoire Royal de Belgique dont la compétence dans l'établissement des programmes de calcul sur ordinateur (I.B.M. 1620) et le dévouement à l'exécution des nombreux calculs et graphiques réclamés par cette recherche, ont permis de la présenter à l'occasion de ce Colloque.

* Is this orbital really necessary. - Publ. Astron. Soc. Pacific. 74, 1962, p. 297. 